\title{
Compulsive Health-Related Internet Use and Cyberchondria
}

\author{
Yasser Khazaala,b,c Anne Chatton ${ }^{d}$ Lucien Rochat ${ }^{\mathrm{e}}$ Vincent Hede $^{\mathrm{d}}$ \\ Kirupamani Viswasam $^{f}$ Louise Penzenstadler ${ }^{d}$ David Berle ${ }^{g, h}$ \\ Vladan Starcevic ${ }^{i}$
}

${ }^{a}$ Addiction Medicine, Department of Psychiatry, Lausanne University Hospital, Lausanne, Switzerland; baculty of Biology and Medicine, University of Lausanne, Lausanne, Switzerland; 'Research Centre, University Institute of

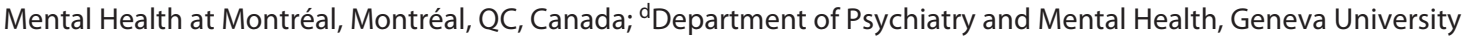
Hospitals, Geneva, Switzerland; ' $F$ Faculty of Psychology, University of Geneva, Geneva, Switzerland; 'Department of Psychiatry, Nepean Hospital, Penrith, NSW, Australia; ${ }^{9}$ Discipline of Clinical Psychology, University of Technology Sydney, Sydney, NSW, Australia; hSchool of Psychiatry, University of New South Wales, Sydney, NSW, Australia; 'Discipline of Psychiatry, Sydney Medical School, Nepean Clinical School, Faculty of Medicine and Health, University of Sydney, Sydney, NSW, Australia

\section{Keywords}

Cyberchondria $\cdot$ Health anxiety $\cdot$ Compulsive Internet use · Internet addiction

\footnotetext{
Abstract

Background: Cyberchondria denotes excessive and repeated online health-related searches associated with an increase in health anxiety. Such searches persist in those with cyberchondria, despite the negative consequences, resembling a pattern of compulsive Internet use. Objectives: The aim of the present study was to assess compulsive healthrelated Internet use in relation to cyberchondria while controlling for related variables. Method: Adult participants $(N=749)$ were recruited from an online platform. They completed questionnaires assessing the severity of cyberchondria (via the Cyberchondria Severity Scale [CSS]), compulsive Internet use adapted for online health-related seeking (via the adapted Compulsive Internet Use Scale [CIUS]), and lev-
}

$\begin{array}{ll}\text { karger@karger.com } & \begin{array}{l}\text { (C) } 2020 \text { The Author(s) } \\ \text { Published by S. Karger AG, Basel }\end{array} \\ \text { Kww.karger.com/ear } & \begin{array}{l}\text { This is an Open Access article licensed under the Creative Commons } \\ \text { Attribution-NonCommercial-4.0 International License (CC BY-NC) } \\ \text { (http://www.karger.com/Services/OpenAccessLicense), applicable to } \\ \text { the online version of the article only. Usage and distribution for com- } \\ \text { mercial purposes requires written permission. }\end{array}\end{array}$

els of intolerance of uncertainty and anxiety, as well as depressive, somatic, and obsessive-compulsive symptoms. A logistic regression analysis was carried out to identify predictors of scores above a cutoff value on the CIUS, indicating compulsive health-related Internet use. Results: The regression output showed that only the CSS total score and sex made a unique, statistically significant contribution to the model, leading to the correct classification of $78.6 \%$ of the cases. Of the CSS subscales, compulsion and distress were the most strongly associated with compulsive health-related Internet use. Conclusions: The finding that the adapted CIUS scores are associated with cyberchondria indicates that cyberchondria has a compulsive component, at least in terms of health-related Internet use. It also suggests that compulsive health-related Internet use persists despite the distress associated with this activity. Males may engage in cyberchondria more compulsively than females. These findings have implications for research and clinical practice.

(c) 2020 The Author(s)

Published by S. Karger AG, Basel

Yasser Khazaal

Addiction Medicine, Department of Psychiatry, Lausanne University Hospital Lausanne University

Bugnon 23a, CH-1011 Lausanne (Switzerland)

yasser.khazaal@chuv.ch 


\section{Introduction}

For many people, the Internet is the main source of information on health [1-3]. Studies in the USA and Europe showed that more than $70 \%$ of Internet consumers conducted online health-related searches [4-6]. The most common reasons for visiting medical websites are seeking advice or information on symptoms, medical conditions, or treatments $[7,8]$ and discussing health concerns with others [9]. Presence of somatic symptoms is particularly associated with recent health-related Internet searches [10]. The feeling of anonymity on the Internet [11] may facilitate searches, queries, and discussions about stigmatized topics [12-14].

The growing availability of medical information on the Internet may help empower people and patients [1, 15-17]. However, health-related information on the Internet varies greatly in quality [18-21] and is not typically tailored to users' needs. Laypersons have few supports and tools to evaluate the quality and credibility of such complex information [22, 23]. Regardless of the possible benefits to the patient-doctor relationship [24] and shared decision-making processes, it is uncommon for people (fewer than 1 in 3 ) to discuss Internet-derived health information with caregivers [7, 25].

In this context, studies have shown that searching for health information may increase levels of distress and uncertainty about one's feared condition in about a third of users [26-29], particularly in those with health anxiety [30]. People who are distressed about their health tend to search the Internet for health purposes more frequently $[25,30]$ and for longer amounts of time than others do. Ambiguous, conflicting, or inadequate online information may increase uncertainty and distress during and following Internet searches, leading to a further increase in search behaviour in an attempt to obtain reassurance [27, 31]. Such reassurance seeking may in turn contribute to further anxiety.

Increased anxiety associated with excessive or repeated health-related Internet searches is called cyberchondria $[32,33]$. The phenomenon denotes a distressing and problematic behaviour rather than a diagnostic entity [34]. It can be time-consuming $[28,33]$ and may lead to functional impairment [26] and increased healthcare use [35]. Cyberchondria and health anxiety are closely related but nonetheless distinct constructs, as cyberchondria also occurs in people without a previously heightened health anxiety $[35,36]$.

Online searches arising from the feeling of uncertainty and the continuation of such searches until a feeling of (transient) reassurance is obtained suggest possible links between cyberchondria and intolerance of uncertainty and obsessive-compulsive disorder (OCD) symptoms. These relationships were demonstrated in previous studies $[31,36-38]$. Fewer studies have explored the association between cyberchondria and anxiety (other than health anxiety) and depressive symptoms. However, medium-strength correlations between scores on the Cyberchondria Severity Scale (CSS) and measures of depressive symptoms have been observed [39].

Cyberchondria also appears to have compulsive features, the behaviour persisting despite its negative effects (i.e., searches are time-consuming and result in increasing distress, neglect of commitments, conflicts with others, difficulties with healthcare providers, and increasing use of health services) [28, 32, 33]. In terms of this persistence in the face of negative consequences, cyberchondria may be associated with compulsive Internet use. Both constructs seem to share an inability to control Internet use despite persisting negative consequences $[29,40]$. As repeatedly demonstrated for compulsive Internet use, coping motives (e.g., using the Internet to escape from negative emotions) [41-44] may also play a role in cyberchondria as a form of reassurance seeking and avoidance behaviour.

Such similarities notwithstanding, only a few studies have assessed the links between cyberchondria and problematic Internet use $[29,45]$. These studies found that cyberchondria was strongly associated with compulsive Internet use, independently from factors such as negative affect or health anxiety [45]. People who reported increased health anxiety following Internet searches for medical information exhibited a significantly greater compulsive Internet use than others did [29]. These studies assessed Internet use behaviour by using scales such as the Compulsive Internet Use Scale (CIUS) [46] and the Problematic Internet Use Questionnaire [47]. The instruments were administered to participants in their original form (e.g., "Do you continue to use the Internet despite your intention to stop?"), leaving open the possibility that the associations found in such studies could have been a consequence of the association between cyberchondria and a general pattern of compulsive Internet use rather than a pattern of compulsive health-related Internet use. Therefore, it is possible that people with cyberchondria use the Internet excessively for other purposes as well [32].

To the best of our knowledge, specific links between cyberchondria and compulsive health-related Internet use have not been studied. Therefore, the aim of the present study was to assess compulsive health-related Internet 
use in relation to cyberchondria while taking into account other relevant constructs (OCD symptoms, intolerance of uncertainty, depressive mood, anxiety, and somatic symptoms) and age, sex, and education. In view of the aforementioned preliminary findings, we hypothesized that there is a strong relationship between cyberchondria and compulsive health-related Internet use.

\section{Method}

\section{Participants and Procedure}

Participants for this study were enrolled from an Internet crowdsourcing platform called Prolific (https://www.prolific.ac/). This platform is specifically dedicated to research studies, and individuals who are interested in participating in research studies register with the platform to be informed of research opportunities [48]. The study was promoted entirely within the Prolific platform and participants were reimbursed for their time at the rate of approximately 5 GBP per person. Both the Nepean Blue Mountains Local Health District Human Research Ethics Committee (LNR/17/NEPEAN/88) in Australia and the Cantonal Research Ethics Committee, Geneva (201800055), in Switzerland approved the study protocol.

Informed consent was obtained from the participants online. They were compensated for their participation directly through the Prolific platform.

Participants were English-speaking persons who were at least 18 years of age from the following countries: Australia, Canada, Ireland, New Zealand, the UK, and the USA. To be included in the present study, participants had to report that they searched for health-related information online during the previous 3 months. The recruitment procedure led to the inclusion of 749 participants in the study analyses.

\section{Measures}

The following self-report measures were administered online.

The Cyberchondria Severity Scale

The CSS [33] is a 33-item questionnaire that aims to assess the severity of cyberchondria. Items (e.g., "I enter the same symptoms into a web search on more than 1 occasion"; "I start to panic when I read online that a symptom I have is found in a rare/serious condition") are rated on a 5-point Likert scale from 1 (never) to 5 (always). The severity of cyberchondria is reported by using the total score derived from 33 items. Previous exploratory factor analyses reported 5 components (distress, compulsion, excessiveness, reassurance, and mistrust of medical professionals) [33]. The internal consistency (Cronbach's $\alpha$ ) of the CSS in the present sample was 0.95.

The Obsessive-Compulsive Inventory Revised

The Obsessive-Compulsive Inventory Revised (OCI-R) [49] is an 18 -item measure of the severity of the symptoms of OCD. The OCI-R includes subscales for pure obsessions as well as for washing, checking, neutralizing, ordering, and hoarding obsessions and compulsions. Each item is rated on a 5-point Likert scale from 0 (not at all) to 4 (extremely). It was reported that the OCI-R is able to differentiate OCD from anxiety disorders [50]. The internal consistency of the OCI-R in the present sample was 0.93 .
The Intolerance of Uncertainty Scale, 12-Item Version

The Intolerance of Uncertainty Scale (IUS) [51] measures intolerance of uncertainty. The 12 items are rated on a 5-point Likert scale from 1 (not at all characteristic of $m e$ ) to 5 (entirely characteristic of me). The scale has 2 factors/subscales of intolerance of uncertainty: inhibitory (e.g., "When I am uncertain I can't function very well") and prospective (e.g., "It frustrates me not having all the information I need"). In this study, the correlation between the 2 subscale scores was $0.76(p<0.01)$, suggesting a high level of shared variance. We therefore included total IUS-SF scores in the analyses. The internal consistency of the IUS-SF in the present sample was 0.93 .

The Patient-Reported Outcomes Measurement Information System Emotional Distress - Short Form Questionnaire

The Patient-Reported Outcomes Measurement Information System Emotional Distress - Short Form Questionnaire (PROMIS) [52] includes 7 items that measure symptoms of anxiety. The items are rated on a 5-point Likert scale from 1 (never) to 5 (always). Scores on the PROMIS correlated strongly with other measures of anxiety [52]. The internal consistency of the PROMIS in the present sample was 0.94 .

\section{The Patient Health Questionnaire-9}

The Patient Health Questionnaire-9 (PHQ-9) [53] is a 9-item assessment tool that measures depressive symptoms. Items are rated on a 4-point Likert scale from 0 (not at all) to 3 (nearly every day). The PHQ-9 showed good convergent validity in comparison to other self-report measures of depressive symptoms [54]. The internal consistency of the PHQ-9 in the present sample was 0.90 .

The Patient Health Questionnaire-15 Somatic Symptom

Severity Scale

The Patient Health Questionnaire-15 (PHQ-15) [55] is an instrument that measures the severity of common somatic symptoms (e.g., stomach pain, dizziness, and headache). Every item relates to a distinct symptom and is scored on a 3-point Likert scale from 0 (not bothered at all) to 2 (bothered a lot). Scores on the PHQ-15 correlated strongly with other measures of somatic symptom distress [55]. The internal consistency of the PHQ-15 in the present sample was 0.83 .

\section{The Compulsive Internet Use Scale}

The CIUS [46] is a 14-item measure of compulsive Internet use. The items are rated on a 5-point Likert scale from 0 (never) to 4 (very often). Item ratings are summed: the higher the score, the more severe the compulsive Internet use. The instrument includes items related to the following components of addictive behaviours: loss of control, preoccupation, withdrawal, conflict, and coping. A single factor solution was retained as the best-fit model in different linguistic samples [56-60]. The internal consistency of the CIUS in the present sample was 0.95 .

The items of the CIUS pertain to the general use of the Internet. To specifically assess health-related Internet use, we modified the questions as shown in the following example: "How often do you find it difficult to stop using the Internet when you are online?" was changed to "How often do you find it difficult to stop using the Internet for health-related purposes when you are online?" The psychometric properties of the CIUS and other Internet addiction scales such as the Internet Addiction Test [61] have been found to 
Table 1. Sample characteristics $(N=749)$

\begin{tabular}{lc}
\hline & Value \\
\hline Age, mean (SD), range, years & $35.38(11.67), 18-75$ \\
PHQ-9, mean (SD), range & $8.42(6.35), 0-27$ \\
PROMIS, mean (SD), range & $18.13(6.80), 7-35$ \\
IUS-SF, mean (SD), range & $32.43(10.556), 12-60$ \\
OCI-R, mean (SD), range & $16.21(13.96), 0-69$ \\
PHQ-15, mean (SD), range & $8.68(5.07), 0-28$ \\
CSS, mean (SD), range & $72.98(22.98), 37-150$ \\
Sex (female), \% & 69.07 \\
Education, \% & 2.13 \\
$\quad$ Not completed high school education (12 years) & 6.53 \\
Completed vocational training & 26 \\
Completed high school education (12 years) & 65.2 \\
Any university-level education (not necessarily completed) & 69.8 \\
CIUS scores, \% & 30.2 \\
$\quad 0-21$ & \\
22-54 & \\
\hline
\end{tabular}

PHQ-9, Patient Health Questionnaire-9; PROMIS, Patient-Reported Outcomes Measurement Information System Emotional Distress - Short Form Questionnaire; IUS-SF, Intolerance of Uncertainty Scale, 12-item version; OCI-R, Obsessive-Compulsive Inventory Revised; PHQ-15, Patient Health Questionnaire-15 Somatic Symptom Severity Scale; CSS, Cyberchondria Severity Scale; CIUS, Compulsive Internet Use Scale.

be maintained [62] when adapted for specific types of Internet use, including Internet gaming, Internet gambling [62], and cybersex $[63,64]$.

\section{Statistical Analyses}

Descriptive statistics were first computed to summarize participants' characteristics. Because of the strong positive asymmetry of the CIUS as a dependent variable, the previously continuous variable had to be categorized after an unsuccessful log transformation. A cutoff of 21 , based on previous studies $[65,66]$, was used in a binary logistic regression with 2 CIUS groups (0: 0-21 score group vs. 1: 22-56). A score $>21$ indicates a compulsive healthrelated Internet use. For all analyses, a $p$ value of 0.05 , two-tailed, was considered significant.

\section{Missing Values}

Of the original 1,048 IP addresses recorded, 27 were duplicates, 25 participants provided no data at all, and 245 did not search for health-related information online during the previous 3 months. Therefore, they were excluded. Of the remaining 751 participants, 2 did not provide their age, leading to a final sample of 749 participants whose data were used in the analyses.

\section{Results}

Participants' characteristics are reported in Table 1. Note that Education has been recoded as a binary categorical variable $(1=$ any university level vs. $0=$ no univer- sity level) for further analyses because participants with a university level of education were overrepresented and participants in the 3 categories with lower educational level were therefore lumped together.

A binary logistic regression was performed on the dichotomized CIUS variable with independent variables including age, education, sex, cyberchondria (CSS), intolerance uncertainty (IUS), obsessive-compulsive symptoms (OCI-R), depressive symptoms (PHQ-9), anxiety symptoms (PROMIS), and somatic symptoms (PHQ-15) to predict group membership. However, when depression (PHQ-9) and anxiety (PROMIS) were entered in the regression as independent variables, signs of multicollinearity appeared, particularly suggested by the strong correlation between the PROMIS and the PHQ-9 ( $r=$ $0.75 ; p<0.001)$. This multicollinearity was also supported by a tolerance score of 0.35 and a variance inflation factor of 2.85 for the PHQ-9, which met the established criteria for multicollinearity (tolerance $<0.40$ and variance inflation factor >2.5) [67]. Consequently, the PHQ-9 was removed from further analyses.

The final analyses indicate that the full model containing age, education, sex, CSS, IUS, OCI-R, PHQ-15, and PROMIS was statistically significant $([\chi 28, N=749]=$ $236.87, p<0.001$ ) over the model with the constant only. The model, as a whole, explained between $27 \%$ (Cox and 
Table 2. Binary logistic regression analysis of 749 participants searching for health information on the Internet

\begin{tabular}{|c|c|c|c|c|c|c|c|c|c|}
\hline Outcome & Predictor & $\beta$ & $\mathrm{SE}_{\beta}$ & $\begin{array}{l}\chi^{2} \\
\text { (Wald's) }\end{array}$ & df & $p$ value & $\begin{array}{l}\mathrm{e}^{\beta} \\
\text { (odds ratio) }\end{array}$ & \multicolumn{2}{|c|}{$95 \%$ CI for $\mathrm{e}^{\beta}$} \\
\hline \multirow[t]{6}{*}{ (low vs. high) } & Education (below university level) & 0.16 & 0.20 & 0.62 & 1 & 0.43 & 1.17 & 0.79 & 1.74 \\
\hline & Sex (male) & 0.53 & 0.21 & 6.43 & 1 & $<0.05$ & 1.69 & 1.13 & 2.54 \\
\hline & CSS & 0.06 & 0.00 & 91.45 & 1 & $<0.001$ & 1.06 & 1.05 & 1.07 \\
\hline & PHQ-15 & -0.03 & 0.03 & 1.19 & 1 & 0.28 & 0.97 & 0.92 & 1.02 \\
\hline & PROMIS & 0.03 & 0.02 & 2.19 & 1 & 0.14 & 1.03 & 0.99 & 1.08 \\
\hline & Constant & -5.86 & 0.65 & 81.94 & 1 & 0.00 & 0.00 & - & - \\
\hline
\end{tabular}

Regarding education and sex, the group of reference was participants with "any university level” and female, respectively. CIUS, Compulsive Health-Related Internet Use Scale; CSS, Cyberchondria Severity Scale; IUS-SF, Intolerance of Uncertainty Scale, 12 -item version; OCI-R, Obsessive-Compulsive Inventory Revised; PHQ-15, Patient Health Questionnaire-15 Somatic Symptom Severity Scale; PROMIS, Patient-Reported Outcomes Measurement Information System Emotional Distress - Short Form Questionnaire; CIUS, Compulsive Internet Use Scale.

Snell) to $38 \%$ (Nagelkerke $R$ squared) of the variance in the CIUS status and correctly classified $78.8 \%$ of the cases. The regression output (Table 2) showed that only two of the independent variables, namely, cyberchondria and sex made a unique, statistically significant contribution to the model. In other words, more prominent cyberchondria symptoms and male sex were both associated with an increased risk of belonging to the high CIUS group.

As it can be argued that a partial overlap in the content between the compulsion subscale of the CSS (which consists of items assessing interference that results from excessive online health information seeking) and the CIUS (which includes items assessing loss of control, preoccupation, withdrawal, conflict, and coping as components of addictive behaviours) may account for their relationship, we fit a new logistic model after removing the compulsion subscale score from the total score of the CSS. The results indicated that cyberchondria still remained a strong and significant predictor of the CIUS status, $\exp (\beta 1)=1.07$, with $95 \%$ CI $[1.06 ; 1.09], p<0.001$.

There was also a partial overlap between item 5 of the CIUS ("lack of sleep due to using the Internet for healthrelated purposes") and item 31 of the CSS ("troubles getting to sleep after researching symptoms or perceived medical conditions online, as the findings play on my mind"). Therefore, we conducted another binary logistic regression analysis after removing the score on item 31 from the CSS. This analysis showed that cyberchondria still remained a strong and significant predictor of the
CIUS status, $\exp (\beta 1)=1.08$, with 95\% CI $[1.06 ; 1.10], p<$ 0.001 .

Considering that there was a strong multicollinearity when simultaneously including various subscales of the CSS as independent predictors in the regression analyses, it was not possible to examine which subscales of the CCS best predicted the CIUS status. Consequently, we examined the association between the CIUS status and subscales of the CSS with point-biserial correlation analyses. The results showed that compulsion $(r=0.52, p<0.001)$, distress $(r=$ $0.50, p<0.001)$, excessiveness $(r=0.40, p<0.001)$ reassurance $(r=0.38, p<0.001)$, and to a lesser extent mistrust $(r=0.13, p<0.001)$ were all significantly and positively associated with compulsive health-related Internet use.

\section{Discussion}

The aim of the present study was to assess compulsive health-related Internet use in relation to cyberchondria and other variables. In contrast to previous studies, which assessed compulsive general Internet use [29, 45], our study specifically assessed compulsive health-related Internet use. This provided confidence that our results were not related to other, non-health-related forms of compulsive Internet use (e.g., those involving social networking, gaming, or watching pornography) [32]. In addition, to avoid potential circularity due to the moderate to strong link commonly reported between health anxiety and cy-
Eur Addict Res 2021;27:58-66 DOI: $10.1159 / 000510922$
Khazaal/Chatton/Rochat/Hede/ Viswasam/Penzenstadler/Berle/Starcevic 
berchondria [35], we included an assessment of general anxiety rather than a measure of health anxiety.

The main finding of the study is that cyberchondria, as assessed by the CSS, significantly predicted compulsive health-related Internet use, as measured by the modified CIUS, while other relevant factors frequently associated with cyberchondria, such as OCD symptoms, intolerance of uncertainty [31,36-38] and anxiety, and somatic symptoms [39] were controlled for. This result held true even when the score on the compulsion subscale of the CSS and a partially overlapping item from the distress subscale of the CSS were removed from the total score of the CSS. This finding is concordant with previous reports showing an association between cyberchondria and compulsive Internet use $[29,45]$. Furthermore, the adaptation of the CIUS for health-related Internet use supports the specific link between cyberchondria and compulsive patterns of health-related Internet use.

Among the CSS subscales, compulsion (e.g., "Researching symptoms or perceived medical conditions online interrupts my online leisure activities") and distress (e.g., "I have trouble relaxing after researching symptoms of perceived medical conditions online") were the most strongly associated with compulsive health-related Internet use. While a strong relationship between the compulsion subscale of the CSS and compulsive health-related Internet use could be expected, a finding that emotional and bodily distress related to excessive online health information seeking and compulsive health-related Internet use is novel and particularly important. This is because it supports the key notion about compulsive healthrelated Internet use, that is, that it persists despite distress associated with this activity. Furthermore, the fact the other dimensions of the CSS, namely, reassurance, excessiveness and mistrust, also positively and significantly correlated with compulsive health-related Internet use suggests that various other aspects of cyberchondria contribute to the outcome as well.

The present study also showed that males were more at risk for compulsive health-related Internet use compared to females. To the extent that there is a close relationship between compulsive and addictive Internet use, this finding is in agreement with the usually higher involvement of men in addictive behaviours [68, 69]. Further research should focus more on these potential differences, as that could also reveal different behavioural patterns and mechanisms associated with cyberchondria.

The other variables included in the study - age, education, intolerance of uncertainty, OCD symptoms, anxiety, and somatic symptoms - did not significantly relate to compulsive health-related Internet use. As mentioned earlier, several of these variables are associated with cyberchondria. This finding can be interpreted as an insufficient impact of such variables on the shared features of cyberchondria and compulsive Internet use, namely, the compulsive aspect that appears to be common to both. Increasing our understanding of cyberchondria calls for studies of the relationships between motives (in particular those related to coping) for online health information seeking and compulsive health-related Internet use.

The results of the present study have to be considered in light of several limitations. First, our use of an online crowdsourcing recruitment platform may have resulted in possible self-selection biases [70]. Furthermore, we cannot exclude the possibility that participants may have been searching for information on behalf of relatives, rather than regarding their own health. Some participants might have searched for health information online for reasons that are not related to health anxiety or any other psychopathology. For example, some individuals could engage in this activity out of curiosity. In addition, given the high number of participants holding a university degree, it is also possible that searching for symptoms may have occurred due to professional reasons.

Studies which investigate these are warranted. We also note that our sample may not be representative of Internet users worldwide and are possibly closer to the Internet users samples of the respective countries involved in the study at hand, particularly if we consider the high rate of university-level education in our sample. Moreover, concerns about inattentiveness among participants who participate in research studies through crowdsourcing platforms [72] may not be well founded, with some evidence suggesting that this limitation applies in equal measure to other forms of recruitment. Second, as women were overrepresented in the sample, the results should be generalized only with caution. Third, the cross-sectional design of the study did not allow exploration of causal or temporal relationships between variables. Finally, applying a cutoff score for the CIUS may in some respects be artificial or prone to error. However, a cutoff of 21 has been determined by latent class analysis to maximize sensitivity for detection of cases meeting the proposed criteria for the DSM-5 Internet gaming disorder [66].

Study limitations notwithstanding, we conclude that compulsive health-related Internet use is significantly associated with cyberchondria. The study suggests that cyberchondria has a compulsive component, at least regarding health-related Internet use. This compulsivity and its link with addictive propensity have implications 
for future research on cyberchondria with regards to assessment of various processes and factors involved in addictive behaviours, such as cue reactivity and attentional biases [68, 69], prefrontal control [70], decision-making [71], metacognitions [72], and motivation for escape. In terms of clinical and treatment implications, the present study suggests a need to take into account and target a possible addictive component of cyberchondria.

\section{Acknowledgements}

We thank all the participants.

\section{Statement of Ethics}

Informed consent was obtained from the participants online. Both the Nepean Blue Mountains Local Health District Human Research Ethics Committee (LNR/17/NEPEAN/88) in Australia and the Cantonal Research Ethics Committee, Geneva (201800055), in Switzerland approved the study protocol.

\section{Conflict of Interest Statement}

The authors have no conflicts of interest to declare.

\section{Funding Sources}

This research was not funded by any specific grant.

\section{Author Contributions}

Y.K., V.S., D.B., and V.H. were responsible for study design and played an active role in data collection. A.C. and L.R. performed the data analyses. All authors have played an active role in preparation and writing of the manuscript.

\section{References}

1 Prescott J, Mackie L. "You sort of go down a rabbit hole...you're just going to keep on searching": a qualitative study of searching online for pregnancy-related information during pregnancy. J Med Internet Res. 2017; 19:e194.

2 Kalckreuth S, Trefflich F, Rummel-Kluge C. Mental health related internet use among psychiatric patients: a cross-sectional analysis. BMC Psychiatry. 2014;14:368.

3 Villani M, Kovess-Masfety V. How do people experiencing schizophrenia spectrum disorders or other psychotic disorders use the internet to get information on their mental health? Literature review and recommendations. JMIR Ment Health. 2017;4(1):e1.

4 Andreassen HK, Bujnowska-Fedak MM, Chronaki CE, Dumitru RC, Pudule I, Santana $S$, et al. European citizens' use of e-health services: a study of seven countries. BMC Public Health. 2007;7:53.

5 Li J, Theng YL, Foo S. Predictors of online health information seeking behavior: changes between 2002 and 2012. Health Informatics J. 2016;22(4):804-14.

6 Trefflich F, Kalckreuth S, Mergl R, RummelKluge C. Psychiatric patients' internet use corresponds to the internet use of the general public. Psychiatry Res. 2015;226(1):136-41.

7 Khazaal Y, Chatton A, Cochand S, Hoch A, Khankarli MB, Khan R, et al. Internet use by patients with psychiatric disorders in search for general and medical informations. Psychiatr Q. 2008;79(4):301.
8 Shuyler KS, Knight KM. What are patients seeking when they turn to the internet? Qualitative content analysis of questions asked by visitors to an orthopaedics web site. J Med Internet Res. 2003;5(4):e24.

9 Greiner C, Chatton A, Khazaal Y. Online selfhelp forums on cannabis: a content assessment. Patient Educ Couns. 2017;100(10): 1943-50.

10 Berle D, Starcevic V, Khazaal Y, Viswasam K, Hede V, McMullan RD. Relationships between online health information seeking and psychopathology. Gen Hosp Psychiatry. 2020;62:96-7.

11 Frost J, Vermeulen IE, Beekers N. Anonymity versus privacy: selective information sharing in online cancer communities. J Med Internet Res. 2014;16(5):e126.

12 Monney G, Penzenstadler L, Dupraz O, Etter JF, Khazaal Y. Mhealth app for cannabis users: satisfaction and perceived usefulness. Front Psychiatry. 2015;6:120.

13 Khazaal Y, Chatton A, Monney G, Nallet A, Khan R, Zullino D, et al. Internal consistency and measurement equivalence of the cannabis screening questions on the paper-andpencil face-to-face assist versus the online instrument. Subst Abuse Treat Prev Policy. 2015;10:8.

14 Jabson JM, Patterson JG, Kamen C. Understanding health information seeking on the internet among sexual minority people: cross-sectional analysis from the health information national trends survey. JMIR Public Health Surveill. 2017;3(2):e39.
15 van der Vaart R, Drossaert CH, Taal E, Drossaers-Bakker KW, Vonkeman HE, van de Laar MA. Impact of patient-accessible electronic medical records in rheumatology: use, satisfaction and effects on empowerment among patients. BMC Musculoskelet Disord. 2014; 15:102.

16 Lemire M, Sicotte C, Paré G. Internet use and the logics of personal empowerment in health. Health Policy. 2008;88(1):130-40.

17 Lee ST, Lin J. A self-determination perspective on online health information seeking: the internet vs. face-to-face office visits with physicians. J Health Commun. 2016;21(6):71422.

18 Khazaal Y, Chatton A, Zullino D, Khan R. Hon label and discern as content quality indicators of health-related websites. Psychiatr Q. 2012;83(1):15-27.

19 Khazaal Y, Chatton A, Cochand S, Jermann F, Osiek C, Bondolfi G, et al. Quality of webbased information on pathological gambling. J Gambl Stud. 2008;24(3):357-66.

20 Shah A, Paly JJ, Efstathiou JA, Bekelman JE. Physician evaluation of internet health information on proton therapy for prostate cancer. Int J Radiat Oncol Biol Phys. 2013;85(4): e173-7.

21 Zermatten A, Khazaal Y, Coquard O, Chatton A, Bondolfi G. Quality of web-based information on depression. Depress Anxiety. 2010; 27(9):852-8 
22 Khazaal Y, Chatton A, Cochand S, Coquard O, Fernandez S, Khan R, et al. Brief discern, six questions for the evaluation of evidencebased content of health-related websites. $\mathrm{Pa}$ tient Educ Couns. 2009;77(1):33.

23 Sbaffi L, Rowley J. Trust and credibility in web-based health information: a review and agenda for future research. J Med Internet Res. 2017;19(6):e218.

24 Tan SS, Goonawardene N. Internet health information seeking and the patient-physician relationship: a systematic review. J Med Internet Res. 2017;19(1):e9.

25 Muse K, McManus F, Leung C, Meghreblian B, Williams JM. Cyberchondriasis: fact or fiction? A preliminary examination of the relationship between health anxiety and searching for health information on the internet. J Anxiety Disord. 2012;26(1):189-96.

26 Doherty-Torstrick ER, Walton KE, Fallon BA. Cyberchondria: parsing health anxiety from online behavior. Psychosomatics. 2016 57(4):390-400

27 White RW, Horvitz E. Experiences with web search on medical concerns and self diagnosis. AMIA Annu Symp Proc. 2009;2009:696700 .

28 Singh K, Brown RJ. Health-related internet habits and health anxiety in university students. Anxiety Stress Coping. 2014;27(5): 542-54.

29 Fergus TA, Dolan SL. Problematic internet use and internet searches for medical information: the role of health anxiety. Cyberpsychol Behav Soc Netw. 2014;17(12):761-5.

30 Baumgartner SE, Hartmann T. The role of health anxiety in online health information search. Cyberpsychol Behav Soc Netw. 2011; 14(10):613-8

31 Fergus TA. Cyberchondria and intolerance of uncertainty: examining when individuals experience health anxiety in response to internet searches for medical information. Cyberpsychol Behav Soc Netw. 2013;16(10):735-9.

32 Starcevic V, Berle D. Cyberchondria: towards a better understanding of excessive health-related internet use. Expert Rev Neurother. 2013;13(2):205-13

33 McElroy E, Shevlin M. The development and initial validation of the cyberchondria severity scale (css). J Anxiety Disord. 2014;28(2): $259-65$.

34 Starcevic V. Cyberchondria: challenges of problematic online searches for health-related information. Psychother Psychosom. 2017;86(3):129-33.

35 Mathes BM, Norr AM, Allan NP, Albanese BJ, Schmidt NB. Cyberchondria: overlap with health anxiety and unique relations with impairment, quality of life, and service utilization. Psychiatry Res. 2018;261:204-11.

36 Fergus TA, Russell LH. Does cyberchondria overlap with health anxiety and obsessivecompulsive symptoms? An examination of latent structure and scale interrelations. J Anxiety Disord. 2016;38:88-94.
37 Fergus TA. Anxiety sensitivity and intoler ance of uncertainty as potential risk factors for cyberchondria: a replication and extension examining dimensions of each construct. J Affect Disord. 2015;184:305-9.

38 Norr AM, Oglesby ME, Raines AM, Macatee RJ, Allan NP, Schmidt NB. Relationships between cyberchondria and obsessive-compulsive symptom dimensions. Psychiatry Res. 2015;230(2):441-6.

39 Barke A, Bleichhardt G, Rief W, Doering BK. The cyberchondria severity scale (css): German validation and development of a short form. Int J Behav Med. 2016;23(5):595-605.

40 Billieux J, van Rooij AJ, Heeren A, Schimmenti A, Maurage P, Edman J, et al. Behavioural addiction open definition 2.0-using the open science framework for collaborative and transparent theoretical development. Addiction. 2017;112(10):1723-4.

41 Franc E, Khazaal Y, Jasiowka K, Lepers T, Bianchi-Demicheli F, Rothen S. Factor structure of the cybersex motives questionnaire. J Behav Addict. 2018;7(3):601-9.

42 Brand M, Laier C, Young KS. Internet addiction: coping styles, expectancies, and treatment implications. Front Psychol. 2014;5 1256.

43 Khazaal Y, Breivik K, Billieux J, Zullino D, Thorens G, Achab S, et al. Game addiction scale assessment through a nationally representative sample of young adult men: item response theory graded-response modeling. J Med Internet Res. 2018;20(8):e10058.

44 Kiraly O, Urban R, Griffiths MD, Agoston C, Nagygyorgy K, Kokonyei G, et al. The mediating effect of gaming motivation between psychiatric symptoms and problematic online gaming: an online survey. J Med Internet Res. 2015; 17:e88.

45 Fergus TA, Spada MM. Cyberchondria: examining relations with problematic internet use and metacognitive beliefs. Clin Psychol Psychother. 2017;24:1322-30.

46 Meerkerk GJ, Van Den Eijnden RJ, Vermulst AA, Garretsen HF. The compulsive internet use scale (cius): some psychometric properties. Cyberpsychol Behav. 2009;12(1):1-6.

47 Demetrovics Z, Szeredi B, Rózsa S. The threefactor model of internet addiction: the development of the problematic internet use questionnaire. Behav Res Methods. 2008;40(2): 563-74.

48 Palan S, Schitter C. Prolific.ac: a subject pool for online experiments. J Behav Exp Finance. 2018;17:22-7.

49 Foa EB, Huppert JD, Leiberg S, Langner R, Kichic R, Hajcak G, et al. The obsessive-compulsive inventory: development and validation of a short version. Psychol Assess. 2002; 14:485-96.

50 Abramowitz JS, Brett JD. Psychometric properties and construct validity of the obsessivecompulsive inventory - revised: replication and extension with a clinical sample. J Anxiety Disord. 2006;20:1016-35.
51 Carleton RN, Norton MA, Asmundson GJ. Fearing the unknown: a short version of the intolerance of uncertainty scale. J Anxiety Disord. 2007;21(1):105-17.

52 Pilkonis PA, Choi SW, Reise SP, Stover AM, Riley WT, Cella D, et al. Item banks for measuring emotional distress from the patientreported outcomes measurement information system (promis $\left.{ }^{\circledR}\right)$ : depression, anxiety, and anger. Assessment. 2011;18(3):263-83.

53 Kroenke K, Spitzer RL, Williams JB. The phq9: validity of a brief depression severity measure. J Gen Intern Med. 2001;16(9):606-13.

54 Berle D, Moulds ML. An experimental investigation of emotional reasoning processes in depression. Br J Clin Psychol. 2013;52(3): 316-29.

55 Kroenke K, Spitzer RL, Williams JB. The phq15: validity of a new measure for evaluating the severity of somatic symptoms. Psychosom Med. 2002;64(2):258-66.

56 Khazaal Y, Chatton A, Horn A, Achab S, Thorens $G$, Zullino D, et al. French validation of the compulsive internet use scale (cius). Psychiatr Q. 2012;83(4):397.

57 Khazaal Y, Chatton A, Atwi K, Zullino D, Khan R, Billieux J. Arabic validation of the compulsive internet use scale (cius). Subst Abuse Treat Prev Policy. 2011;6:32.

58 Guertler D, Broda A, Bischof A, Kastirke N, Meerkerk GJ, John U, et al. Factor structure of the compulsive internet use scale. Cyberpsychol Behav Soc Netw. 2014;17(1):46-51.

59 Dhir A, Chen S, Nieminen M. Psychometric validation of the Chinese compulsive internet use scale (cius) with taiwanese high school adolescents. Psychiatric Q. 2015;86(4):58196.

60 Meerkerk GJ, Van Den Eijnden RJ, Vermulst AA, Garretsen HF. The compulsive internet use scale (cius): some psychometric properties. Cyberpsychol Behav. 2009;12(1):1-6.

61 Young KS. Caught in the net. New York: John Wiley \& Sons; 1998.

62 Khazaal Y, Achab S, Billieux J, Thorens G, Zullino D, Dufour M, et al. Factor structure of the internet addiction test in online gamers and poker players. JMIR Ment Health. 2015; 2(2):e12.

63 Downing MJ Jr, Antebi N, Schrimshaw EW. Compulsive use of internet-based sexually explicit media: adaptation and validation of the compulsive internet use scale (cius). Addict Behav. 2014;39(6):1126-30.

64 Wery A, Deleuze J, Canale N, Billieux J. Emotionally laden impulsivity interacts with affect in predicting addictive use of online sexual activity in men. Compr Psychiatry. 2018;80 192-201.

65 Guertler D, Rumpf HJ, Bischof A, Kastirke N, Petersen KU, John U, et al. Assessment of problematic internet use by the compulsive internet use scale and the internet addiction test: a sample of problematic and pathological gamblers. Eur Addict Res. 2014;20(2):75-81. 
66 Besser B, Rumpf HJ, Bischof A, Meerkerk GJ, Higuchi S, Bischof G. Internet-related disorders: development of the short compulsive internet use scale. Cyberpsychol Behav Soc Netw. 2017;20(11):709-17.

67 Allison P-D, Cary NC. Logistic regression using the sas system: theory and application. Cary, NC: SAS Institute, John Wiley \& Sons; 1999.
68 Khazaal Y, Zullino D, Billieux J. The Geneva smoking pictures: development and preliminary validation. Eur Addict Res. 2012;18(3): 103-9.

69 Billieux J, Khazaal Y, Oliveira S, de Timary P, Edel Y, Zebouni F, et al. The Geneva appetitive alcohol pictures (gaap): development and preliminary validation. Eur Addict Res. 2011; 17(5):225-30.

70 Brand M, Young KS, Laier C. Prefrontal control and internet addiction: a theoretical model and review of neuropsychological and neuroimaging findings. Front Hum Neurosci. 2014;8:375.
71 Bechara A, Damasio H. Decision-making and addiction (part I): impaired activation of somatic states in substance dependent individuals when pondering decisions with negative future consequences. Neuropsychologia. 2002;40(10): 1675-89.

72 Spada MM, Caselli G, Nikčević AV, Wells A. Metacognition in addictive behaviors. Addict Behav. 2015;44:9-15. 